\title{
The hemodynamic effects of norepinephrine: far more than an increase in blood pressure!
}

\author{
Pierre Foulon, Daniel De Backer \\ Department of Intensive Care, CHIREC Hospitals, Université Libre de Bruxelles, Brussels, Belgium \\ Correspondence to: Dr Daniel De Backer. Department of Intensive Care, CHIREC hospitals, Boulevard du Triomphe 201, B-1160 Brussels, Belgium. \\ Email: ddebacke@ulb.ac.be. \\ Provenance: This is an invited Editorial commissioned by Section Editor Guo-wei Tu (Department of Critical Care Medicine, Zhongshan Hospital, \\ Fudan University, Shanghai, China). \\ Comment on: Hamzaoui O, Jozwiak M, Geffriaud T, et al. Norepinephrine exerts an inotropic effect during the early phase of human septic shock. Br \\ J Anaesth 2018;120:517-24.
}

Submitted Aug 31, 2018. Accepted for publication Sep 12, 2018.

doi: $10.21037 /$ atm.2018.09.27

View this article at: http://dx.doi.org/10.21037/atm.2018.09.27

Vasopressors are commonly used to correct hypotension. Among these, norepinephrine is the preferred first line vasopressor. Compared to dopamine, norepinephrine improves outcome in patients with septic shock (1) and in cardiogenic shock (2). Recently a trial comparing norepinephrine to ephedrine boluses in peri-operative period, demonstrated that norepinephrine was associated with lower occurrence of postoperative organ dysfunction (3). Compared to epinephrine, norepinephrine administration in patients with cardiogenic shock improved shock resolution and was associated to a trend in a lower mortality (4). During shortage of norepinephrine in the US, substitution of norepinephrine was associated with a transient increase in mortality rate in septic shock patients (5). Thus, norepinephrine is the vasopressor agent of choice, but what are the hemodynamic effects of norepinephrine beyond increasing blood pressure?

Interestingly, it has been demonstrated in different trials that correction of hypotension with administration of norepinephrine at early stages of shock is associated with an increase in cardiac output. Intuitively, one may expect that raising blood pressure, and thus afterload, would rather be associated with a decrease in cardiac output. What mechanisms could be associated with this increase in cardiac output? Theoretically norepinephrine may act on the four determinants of cardiac output (6): heart rate, preload, contractility and afterload. As norepinephrine minimal affects heart rate, most of the hemodynamic effects are related to its effects on stroke volume.

First norepinephrine markedly affect preload. In 25 septic shock patients who were fluid responsive, as defined by a positive passive leg raising test, norepinephrine increased central venous pressure by $23 \%$, left ventricular end-diastolic area by $9 \%$ and global end diastolic volume by $9 \%$ (7). This effect on preload was accompanied by an $11 \%$ increase in cardiac index. Venoconstriction is the mechanism by which norepinephrine increases preload, redistributing blood from unstressed to stressed volume. This hypothesis was confirmed by the demonstration that norepinephrine increased mean systemic pressure, an estimate of the distending pressure in the small veins and venules, which contain most of the blood in the body (8). As mean systemic pressure increased without altering right atrial pressure, the gradient for venous return (and thus cardiac output) increased. Of note, norepinephrine also hindered somewhat the gradient for venous return by increasing venous vascular resistances, so that a greater mean systemic pressure is required to preserve venous return.

Interestingly, norepinephrine decreases the need for volume replacement (9). The sepsis induced vasodilation also affects capacitance veins. Hence a large amount of volume is required to refill the tank. Norepinephrine also restore vascular tone in capacitance veins, thereby decreasing amount of fluids needed to restore the gradient for venous return. There are nevertheless two negative counterparts to the use of norepinephrine. On the one hand, excessive vasoconstriction is not desired, and the aim 
of norepinephrine use in sepsis should be to restore vascular tone in the context of venous (and arterial) dilation, not to substitute to fluid replacement in the context of true hypovolemia. Importantly, sepsis associated hypovolemia is not only related to blood redistribution between venous compartments but also due to a true hypovolemic component related to external losses (diarrhea, sweating...) or to capillary leak. On the other hand, norepinephrine can also promote capillary leak as it increases hydrostatic pressure together with the increase in venous resistances.

A recent finding confirms that norepinephrine also increases contractility. In 38 patients with septic shock, Hamzaoui et al. (10) observed that norepinephrine administration increased left ventricular ejection fraction, stroke volume, anterior displacement of mitral and tricuspid annulus. These effects were also observed in the subgroup of patients with impaired contractility. These effects seem independent of any effect of preload. As the patients were adequately fluid resuscitated at inclusion, there were minimal effects on preload (minimal changes in mitral inflow pattern or left ventricular end-diastolic area). The potential mechanisms associated with this increase in contractility were discussed in an accompanying editorial (11). These encompass an inotropic effect directly related to the (limited) beta-adrenergic stimulation as well as to the increase in coronary artery perfusion pressure related to the increase in diastolic pressure. The combination of the rise in arterial pressure and contractility may improve ventriculoarterial coupling. In patients with septic shock, hypotension is often associated with a decreased arterial elastance, which in turn leads to reduced ventriculo-arterial coupling (12). The correction of hypotension with norepinephrine increases arterial elastance (10) which, associated with an increase in contractility may improve ventriculo-arterial coupling. Interestingly, Guinot et al. (13) reported that stroke volume increased only in patients with impaired ventriculo-arterial coupling at baseline and who improved their ventriculo-arterial coupling during norepinephrine administration, even if norepinephrine corrected arterial hypotension in all patients.

The impact of norepinephrine on left ventricular afterload should not be neglected. While correction of hypotension has many beneficial effects as reported above, in some patients the increase in blood pressure is represent an exaggerated cardiac afterload that the heart can hardly overcome. It seems that the impact on cardiac output would depend on the balance between the potentially beneficial effects (improve ventriculo-arterial coupling; increase coronary artery perfusion...) and the impact on afterload (especially if myocardial function is altered).

An important aspect to recall is that several of these effects may vanish over time. The impact of the increase in preload is likely to be time-dependent. Indeed, while preload-dependency is frequent at early stages of sepsis, it becomes less common at later stages. Even if norepinephrine similarly affects mean systemic pressure at later stages in sepsis, this would not result in an increase in cardiac index when the patient is preload-independent. The increase in venous resistance may even slightly impair cardiac index. Similarly, the effect of norepinephrine on contractility may disappear over time, due to down regulation of beta-adrenergic receptors (14). The impact of raising afterload would remain identical over time or can even be more dramatic if the heart performances exhaust with time. Indeed, one third of the case of myocardial depression became evident only after 24-48 h of support with norepinephrine (15).

In addition to these systemic hemodynamic effects, norepinephrine also exerts regional hemodynamic effects that should be highlighted. Compared to epinephrine, norepinephrine is associated with a better balance between oxygen delivery and oxygen consumption in the splanchnic area (16). Similarly, the other alternatives like phenylephrine or vasopressin derivatives have all be shown to be associated with worse splanchnic hemodynamics $(17,18)$. The impact of norepinephrine on the microcirculation may be more variable. While vasopressors, including norepinephrine, intrinsically decrease microvascular perfusion by increasing precapillary sphincter tone, vasopressors may also improve organ perfusion by restoring perfusion pressure. Accordingly, the net effect on microvascular perfusion is quite variable, with neutral effects, improvements or alterations in microvascular perfusion being all observed in various patients with septic shock (19-21). Baseline blood pressure and microvascular perfusion seem to affect the response to norepinephrine, with improvements in microvascular perfusion when one of these is altered at baseline. Given this variable effect, it sounds reasonable to evaluate these effects during norepinephrine administration, especially considering the relationship between severity and duration of microvascular alterations and outcome in patients with sepsis $(22,23)$.

In conclusion, norepinephrine appears to have interesting hemodynamic properties (Figure 1) in addition to its impact on arterial pressure, especially at the early stages of septic shock, but norepinephrine should not be used as a surrogate 


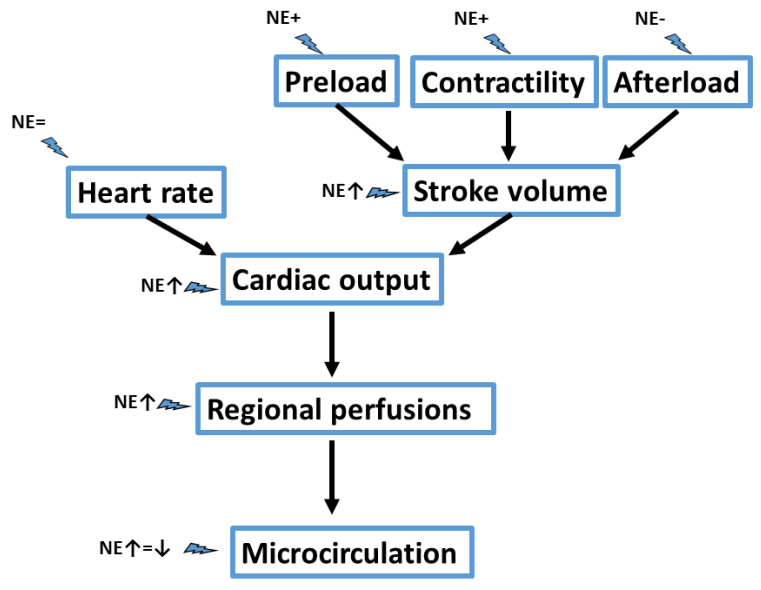

Figure 1 Summary of the various hemodynamic effects of norepinephrine. NE, denotes norepinephrine.

for volume replacement or as an inotropic agent. Evaluation of fluid responsiveness, cardiac function and tissue perfusion should be regularly performed in patients receiving norepinephrine.

\section{Acknowledgments}

None.

\section{Footnote}

Conflicts of Interest: The authors have no conflicts of interest to declare.

\section{References}

1. De Backer D, Aldecoa C, Njimi H, et al. Dopamine versus norepinephrine in the treatment of septic shock: a metaanalysis. Crit Care Med 2012;40:725-30.

2. De Backer D, Biston P, Devriendt J, et al. Comparison of dopamine and norepinephrine in the treatment of shock. N Engl J Med 2010;362:779-89.

3. Futier E, Lefrant JY, Guinot PG, et al. Effect of Individualized vs Standard Blood Pressure Management Strategies on Postoperative Organ Dysfunction Among High-Risk Patients Undergoing Major Surgery: A Randomized Clinical Trial. JAMA 2017;318:1346-57.

4. Levy B, Clere-Jehl R, Legras A, et al. Epinephrine Versus Norepinephrine for Cardiogenic Shock After Acute
Myocardial Infarction. J Am Coll Cardiol 2018;72:173-82.

5. Vail E, Gershengorn HB, Hua M, et al. Association Between US Norepinephrine Shortage and Mortality Among Patients With Septic Shock. JAMA 2017;317:1433-42.

6. Vincent JL, De Backer D. ICU nephrology: the implications of cardiovascular alterations in the acutely ill. Kidney Int 2012;81:1060-6.

7. Monnet X, Jabot J, Maizel J, et al. Norepinephrine increases cardiac preload and reduces preload dependency assessed by passive leg raising in septic shock patients. Crit Care Med 2011;39:689-94.

8. Persichini R, Silva S, Teboul JL, et al. Effects of norepinephrine on mean systemic pressure and venous return in human septic shock. Crit Care Med 2012;40:3146-53.

9. Perner A, De Backer D. Understanding hypovolaemia. Intensive Care Med 2014;40:613-5.

10. Hamzaoui O, Jozwiak M, Geffriaud T, et al. Norepinephrine exerts an inotropic effect at the early phase of human septic shock. Br J Anaesth 2018;120:517-24.

11. De Backer D, Pinsky M. Norepinephrine improves cardiac function during septic shock, but why? Br J Anaesth 2018;120:421-4.

12. Guarracino F, Ferro B, Morelli A, et al. Ventriculoarterial decoupling in human septic shock. Crit Care 2014;18:R80.

13. Guinot PG, Longrois D, Kamel S, et al. VentriculoArterial Coupling Analysis Predicts the Hemodynamic Response to Norepinephrine in Hypotensive Postoperative Patients: A Prospective Observational Study. Crit Care Med 2018;46:e17-25.

14. Silverman HJ, Penaranda R, Orens JB, et al. Impaired beta-adrenergic receptor stimulation of cyclic adenosine monophosphate in human septic shock: association with myocardial hyporesponsiveness to catecholamines. Crit Care Med 1993;21:31-9.

15. Vieillard-Baron A, Caille V, Charron C, et al. Actual incidence of global left ventricular hypokinesia in adult septic shock. Crit Care Med 2008;36:1701-6.

16. De Backer D, Creteur J, Silva E, et al. Effects of dopamine, norepinephrine, and epinephrine on the splanchnic circulation in septic shock: Which is best? Crit Care Med 2003;31:1659-67.

17. Reinelt H, Radermacher P, Kiefer P, et al. Impact of exogenous beta-adrenergic receptor stimulation on hepatosplanchnic oxygen kinetics and metabolic activity in septic shock. Crit Care Med 1999;27:325-31.

18. Klinzing S, Simon M, Reinhart K, et al. High-dose 
Page 4 of 4

vasopressin is not superior to norepinephrine in septic shock. Crit Care Med 2003;31:2646-50.

19. Thooft A, Favory R, Salgado DR, et al. Effects of changes in arterial pressure on organ perfusion during septic shock. Crit Care 2011;15:R222.

20. Dubin A, Pozo MO, Casabella CA, et al. Increasing arterial blood pressure with norepinephrine does not improve microcirculatory blood flow: a prospective study. Crit Care 2009; 13:R92.

21. Georger JF, Hamzaoui O, Chaari A, et al. Restoring arterial pressure with norepinephrine improves muscle

Cite this article as: Foulon P, De Backer D. The hemodynamic effects of norepinephrine: far more than an increase in blood pressure! Ann Transl Med 2018;6(Suppl 1):S25. doi:10.21037/ atm.2018.09.27

\section{Foulon and De Backer. The hemodynamic effects of norepinephrine}

tissue oxygenation assessed by near-infrared spectroscopy in severely hypotensive septic patients. Intensive Care Med 2010;36:1882-9.

22. Sakr Y, Dubois MJ, De Backer D, et al. Persistant microvasculatory alterations are associated with organ failure and death in patients with septic shock. Crit Care Med 2004;32:1825-31.

23. De Backer D, Donadello K, Sakr Y, et al. Microcirculatory alterations in patients with severe sepsis: impact of time of assessment and relationship with outcome. Crit Care Med 2013;41:791-9. 